\title{
Dynamic Changes in Neutrophil-to-Lymphocyte Ratio are Associated with Survival and Liver Toxicity Following Stereotactic Body Radiotherapy for Hepatocellular Carcinoma
}

\author{
Chih-Weim Hsiang' \\ Wen-Yen Huang ${ }^{2,3}$ \\ Jen-Fu Yang ${ }^{2}$ \\ Po-Chien Shen $\mathbb{D}^{2}$ \\ Yang-Hong Dai ${ }^{2}$ \\ Ying-Fu Wang ${ }^{2}$ \\ Chun-Shu Lin ${ }^{2}$ \\ Wei-Chou Chang (D) \\ Cheng-Hsiang Lo ${ }^{2}$ \\ 'Department of Radiology, Tri-Service \\ General Hospital, National Defense \\ Medical Center, Taipei, Taiwan; \\ ${ }^{2}$ Department of Radiation Oncology, Tri- \\ Service General Hospital, National \\ Defense Medical Center, Taipei, Taiwan; \\ ${ }^{3}$ Institute of Clinical Medicine, National \\ Yang-Ming University, Taipei, Taiwan
}

\begin{abstract}
Purpose: Immune response to antitumor therapies has been correlated with oncologic outcomes. This study aimed to determine whether dynamic changes in immune parameters could predict survival outcomes and assess their relationship with liver toxicity in hepatocellular carcinoma (HCC) patients treated with stereotactic body radiation therapy (SBRT). Methods: Data on pre- and post-SBRT (within 3 months) peripheral blood cell counts, neutrophil-to-lymphocyte ratio (NLR), and platelet-to-lymphocyte ratio (PLR) were retrospectively collected. Kinetic changes in these immune parameters and delta-NLR (dNLR) and deltaPLR (dPLR) in response to SBRT were evaluated. Overall survival (OS) and progression-free survival (PFS) were compared based on baseline NLR/PLR and dNLR/dPLR. Additionally, the association of these dynamic measures with liver toxicity was determined.

Results: The study included 93 patients with a median 10.7-month follow-up. Significant increases in NLR $(p<0.001)$ and PLR $(p=0.003)$ were observed after SBRT. In the multivariable analysis, elevated pre-SBRT NLR $(p<0.001)$ and $\operatorname{dNLR}(p=0.011)$ were predictive of worse OS. dNLR was not associated with PFS. Neither PLR nor dPLR was predictive of survival outcomes. Patients with Child-Turcotte-Pugh class B had higher dNLR and greater risk of liver toxicity than class A counterparts. Receiver operating characteristic curve analysis found that $\mathrm{dNLR} \geq 1.9$ was an optimal cut-off value for determining liver toxicity risk $(35.1 \%$ vs $7.5 \%, p=0.002)$.

Conclusion: Baseline NLR and dNLR can complementarily predict OS in HCC patients treated with SBRT. Elevated dNLR is associated with worse OS and development of liver toxicity, possibly through their relationship with baseline liver function. Dynamic changes in NLR should be monitored in HCC care.
\end{abstract}

Keywords: hepatocellular carcinoma, neutrophil-to-lymphocyte ratio, platelet-tolymphocyte ratio, stereotactic body radiotherapy, liver toxicity

\section{Introduction}

Several prognostic biomarkers for hepatocellular carcinoma (HCC), including tumor number/size, presence of vascular invasion, extrahepatic spread, preserved liver function, patients' performance status, and alpha-fetoprotein (AFP) level, have been well defined. ${ }^{1}$ With the development of immuno-oncology, increasing evidence has suggested a prognostic role of host immune system and cancer-related inflammation in various malignancies, including HCC.,3 Changes in complete blood count (CBC)
Department of Radiation Onsiang Lo

Tri-Service General Hospital, National

Defense Medical Center, No. 325, Sec 2,

Cheng-Gong Road, Neihu, Taipei, I I4,

Taiwan

Tel +886-2-87927I 22

Fax +886-2-66002357

Email Isir183@yahoo.com.tw 
constituents have been linked to an inflammatory state in malignancy, including decreased lymphocyte counts and hemoglobin levels and increased leukocyte and neutrophil counts. In certain patient populations, several inflammatory markers, including neutrophil-to-lymphocyte ratio (NLR), platelet-to-lymphocyte ratio (PLR), and cytokines, have been found to predict survival outcomes, chemotherapy toxicities, and response to antitumor therapies. ${ }^{4}$ Specific for HCC, an elevated pretreatment NLR or PLR indicated poorer survival and early recurrence among patients receiving curative resection, ${ }^{5}$ liver transplantation, ${ }^{6}$ transarterial chemoembolization, ${ }^{7,8}$ radiofrequency ablation (RFA), ${ }^{9}$ and sorafenib. ${ }^{10,11}$ Dynamic changes in inflammatory markers may reflect host immune response to therapy and help predict treatment outcomes. ${ }^{12,13}$ Accordingly, dynamic inflammatory status and host immune response, with other clinicopathologic features, may aid in therapeutic decision-making.

Stereotactic body radiotherapy (SBRT) is an effective local treatment for unresectable or medically inoperable HCC patients. ${ }^{14-16}$ SBRT had greater antitumor efficacy than conventional fractionated radiotherapy, probably through increased expression of various immunostimulatory cytokines within the irradiated tumor microenvironment and activation of anti-tumor T cells, thereby eliciting an enhanced antitumor immunity. ${ }^{17}$ Furthermore, studies on liver and pancreatic cancers suggested that SBRT can preserve lymphocytes, which is critical for antitumor immunity. ${ }^{12,18}$ The prognostic significance of HCC patients' inflammatory states, expressed by NLR, on SBRT outcomes was evaluated. ${ }^{19}$ Elevated pre-SBRT NLR predicted inferior overall survival (OS) and the development of radiationrelated liver toxicity. However, data on host immune response to SBRT in HCC patients and how it relates to treatment outcomes are very limited.

Thus, this study investigated the prognostic value of dynamic changes in immune parameters for predicting survival outcomes of HCC patients after SBRT and the association between peri-SBRT immune changes and liver toxicity.

\section{Materials and Methods}

\section{Patient Selection}

Medical records of all HCC patients treated with SBRT at our institution during December 2007-August 2018 were retrospectively reviewed. The inclusion criteria were HCC confirmed pathologically or based on imaging criteria; Child-Turcotte-Pugh (CTP) class A or B liver function at baseline; and available peripheral CBC with differential at baseline and within 3 months of SBRT. Patients who received prior liver radiotherapy, had other active cancer within 5 years before SBRT, or received preoperative SBRT were excluded. The indications for SBRT for HCC in our institute were previously reported. ${ }^{20}$ The institutional review board of the Tri-Service General Hospital approved this study (permit number: 1-107-05-016) and waived the requirement for informed consent because of the retrospective nature of the study assessed as low risk for patients. All the data are de-identified on collection and analysis. The study was conducted in compliance with the Declaration of Helsinki and other ethical guidelines.

\section{Immune Parameters}

Blood samples drawn at baseline and within 3 months after SBRT were used to compile pre- and post-SBRT immune profiles, which included peripheral leukocyte, neutrophil, lymphocyte, haemoglobin, and platelet counts. When multiple CBC test results were available after SBRT, laboratory values most proximal to the last fraction of SBRT in the absence of clinical evidence of infection were analysed. NLR and PLR, defined as peripheral neutrophil and platelet counts divided by lymphocyte counts, respectively, were calculated for each patient. Delta values were defined as post-SBRT levels minus pre-SBRT levels for delta-NLR (dNLR) and delta-PLR (dPLR). Acute severe lymphopenia (ASL) was defined as lymphocyte count $<500 / \mu \mathrm{L}$ within 3 months of initiating radiotherapy. $^{12}$

\section{SBRT}

For most patients, SBRT for HCC was performed with a CyberKnife radiosurgery system (Accuray, Sunnyvale, CA). Details of the target definition, radiotherapy technique, and treatment planning have been described previously. ${ }^{21}$ After June 2017, patients were treated with Versa HD (Elekta AB, Stockholm, Sweden) using active breathing control (ABC) to manage breathing motion. For cases not tolerating $\mathrm{ABC}$, abdominal compression was performed to reduce liver motion, and internal target volume (ITV) was estimated with four-dimensional computed tomography (CT). The planning target volume was generated with a margin of $0-8 \mathrm{~mm}$ from the gross tumor volume or 0 $5 \mathrm{~mm}$ from ITV, if available. The SBRT regimen was prescribed individually based on normal organ dose constraints as determined by the institutional protocol. 
Patients underwent clinical examination, blood work, and liver triphasic $\mathrm{CT}$ or magnetic resonance imaging at $1-$ 3 months after SBRT and every 3-4 months thereafter, as appropriate. Liver toxicity was defined as declines in CTP score by $\geq 2$ within 3 months after completing SBRT in the absence of intrahepatic progression based on the modified Response Evaluation Criteria in Solid Tumors. ${ }^{22}$ Liver toxicity assessment was censored at the time of subsequent liver-directed therapies. The analysis on liver toxicity was limited to patients with adequate follow-up of $\geq 3$ months or death or development of liver toxicity within 3 months.

\section{Statistical Analysis}

Differences between pre- and post-SBRT immune parameters were evaluated using the Wilcoxon signed-rank test, a nonparametric method for two dependent samples. The Mann-Whitney $U$-test and Fisher's exact test were used to compare continuous and categorical variables between groups, respectively. The Kaplan-Meier method was used to estimate OS and progression-free survival (PFS), which were measured from the first SBRT fraction. Differences between subgroups in survival were assessed using the Log rank test. The association among covariates was assessed using Pearson correlation coefficients for continuous variables or Cramer's V coefficients for categorical variables. Cox proportional hazard model was used to assess the association of immune parameters with survival outcomes. The optimal NLR cut-off value for distinguishing the two groups was established using a receiver operating characteristic curve analysis and Youden index. Variables with $p$-values $<0.1$ in the univariable analyses were subjected to multivariable analysis. Two-tailed $p$-values $<0.05$ were considered statistically significant in all analyses. All analyses were performed using SPSS version 22 software (SPSS Inc, Chicago, IL).

\section{Results}

\section{Patient Characteristics}

Data of $93 \mathrm{HCC}$ patients were analyzed, with median posttreatment follow-ups of 10.7 (interquartile range [IQR], 3.827.7) and 33.1 (IQR, 10.1-101.4) months for all patients and those still alive $(\mathrm{n}=18,19.4 \%)$, respectively (Table 1$)$. The median age was 65 (IQR, 55-74) years; most patients had CTP class A liver function. Most patients had advanced stage HCC (Barcelona Clinic Liver Cancer stage C, 66.7\%). SBRT was delivered in 4-6 fractions, with the median biologically
Table I Patient and Treatment Characteristics $(n=93)$

\begin{tabular}{|c|c|}
\hline \multicolumn{2}{|l|}{ Characteristics } \\
\hline Male, no. (\%) & $72(77.4)$ \\
\hline Age, median (IQR), years & $65(55-74)$ \\
\hline Viral hepatitis, no. (\%) & \\
\hline HBV & $52(55.9)$ \\
\hline $\mathrm{HCV}$ & $26(28.0)$ \\
\hline Both & $5(5.4)$ \\
\hline None & $10(10.8)$ \\
\hline Total GTV, median (IQR), $\mathrm{cm}^{3}$ & $|3| .4(43 .|-3| 4.2)$ \\
\hline PTV, median (IQR), cc & $182.2(70.8-452.5)$ \\
\hline Multiple tumors, no. (\%) & $64(68.8)$ \\
\hline Macrovascular invasion, no. (\%) & $34(36.6)$ \\
\hline Extrahepatic spread, no. (\%) & $25(26.9)$ \\
\hline ECOG performance status, no. (\%) & \\
\hline $0-1$ & $72(77.4)$ \\
\hline$\geq 2$ & $21(22.6)$ \\
\hline AFP level, no. (\%) & \\
\hline$<400 \mathrm{ng} / \mathrm{mL}$ & $53(57.0)$ \\
\hline$\geq 400 \mathrm{ng} / \mathrm{mL}$ & $40(43.0)$ \\
\hline Total bilirubin, median (IQR), mg/dL & $0.8(0.6-1.4)$ \\
\hline Albumin, median (IQR), g/dL & $3.6(3.2-3.9)$ \\
\hline CTP class, no. (\%) & \\
\hline A & $65(69.9)$ \\
\hline B & $28(30.1)$ \\
\hline BCLC stage, no. (\%) & \\
\hline O-A & $14(15.1)$ \\
\hline B & $14(15.1)$ \\
\hline C & $62(66.7)$ \\
\hline $\mathrm{D}$ & $3(3.2)$ \\
\hline SBRT dose, median (range), Gy & $45(25-60)$ \\
\hline Fraction number, median (range) & $5(4-6)$ \\
\hline BED, median (IQR), Gy & $85.5(70.4-100.0)$ \\
\hline Prior liver-directed therapy ${ }^{\dagger}$, no. (\%) & \\
\hline Liver resection & $24(25.8)$ \\
\hline RFA & $13(14.0)$ \\
\hline TACE & $49(52.7)$ \\
\hline Prior treatment number ${ }^{\dagger}$, no (\%) & \\
\hline 0 & $39(41.9)$ \\
\hline $1-3$ & $37(39.8)$ \\
\hline$>3$ & $17(18.3)$ \\
\hline
\end{tabular}

Note: ${ }^{\dagger}$ More than one may apply.

Abbreviations: AFP, $\alpha$-fetoprotein; BCLC, Barcelona Clinic Liver Cancer; BED, biologically effective dose; CTP, Child-Turcotte-Pugh liver function scale; ECOG, Eastern Cooperative Oncology Group; GTV, gross tumor volume; HBV, hepatitis $B$ virus; $\mathrm{HCV}$, hepatitis $\mathrm{C}$ virus; IQR, interquartile range; PTV, planning target volume; RFA, radiofrequency ablation; SBRT, stereotactic body radiotherapy; TACE, trans-arterial chemoembolization. 
effective dose (BED) of 85.5 (IQR, 70.4-100.0) Gy, calculated with an $\alpha / \beta$ of 10 .

\section{Changes in Peri-SBRT Immune Parameters}

The median date of CBC collection was 34 (IQR, 2146) days after SBRT. Immune parameters of all patients are detailed in Table 2. Total leukocyte, neutrophil, lymphocyte, and platelet counts decreased significantly after SBRT (all $p<0.001$ ), with a median (range) change of $-27.5 \%$ ( -72.0 to 139.3$),-22.7 \%$ ( -70.2 to 258.6$)$, $-42.3 \%$ ( -90.3 to 167.5$)$, and $-27.4 \%$ ( -83.2 to 105.9$)$, respectively, while no significant difference was observed for haemoglobin levels. ASL developed in 35 (37.6\%) patients, among whom five had baseline lymphocyte counts $<500 / \mu \mathrm{L}$. Despite similarly decreasing trends in composites after radiotherapy, there was a significant increase in post-SBRT NLR compared with pre-SBRT NLR (mean \pm standard deviation [SD], $5.9 \pm 5.3$ vs $3.6 \pm 2.5, p<0.001$ ), with a median dNLR of 1.3 (range, -10.8 to 23.6 ) and percentage change of $47.6 \%$ ( -81.9 to 894.3$)$. Similar results were obtained for PLR $(190 \pm 180$ vs $132 \pm 79, p=0.003)$, with the median dPLR of 32 (range, -261 to 920) and percentage change of $30.2 \%$ ( -72.9 to 740.7$)$.

\section{Immune Parameters and Survival Outcomes}

The median OS of the entire cohort was 11.5 months $(95 \%$ confidence interval [CI], 6.1-17.0). The 1-, 3-, and 5-year OS rates were $49.6 \%, 24.1 \%$, and $15.5 \%$, respectively. In the univariable analysis, statistically significant associations between worse OS and higher pre-SBRT NLR and dNLR (both treated as a continuous variable) was noted $(p<0.001$ and $p=0.001$, respectively) (Table 3 ). PLR had trends toward significance for OS $(p=0.070)$, but dPLR had no association with OS ( $p=0.401)$.

Cramer's V coefficients among categorical variables were all $<0.3$; thus, their collinearity was not evident. Given the high correlation between NLR and PLR ( $r=0.623$ ), two different Cox proportional hazard models were established to prevent collinearity in the multivariable analysis. Multivariable analysis demonstrated that elevated pre-SBRT NLR (hazard ration [HR], 1.24 ; 95\% CI, $1.12-1.38$ per 1-unit increase; $p<0.001$ ) and dNLR (HR, 1.10; 95\% CI, 1.02-1.18 per 1-unit increase; $p=0.011$ ) were predictive of worse OS. CTP class B and presence of multiple tumors were independent predictors of worse OS in the NLR model. Pre-SBRT PLR was not prognostic of OS in the multivariable analysis.

Using the pre-SBRT NLR cut-off value of 2.4 from our previous study, ${ }^{19}$ the median OS for patients with NLR

Table 2 Dynamic Changes of Complete Blood Counts and Immune Parameters Pre- and Post-SBRT

\begin{tabular}{|c|c|c|c|c|}
\hline Parameter & Pre-SBRT & Post-SBRT & $\%$ Change & $P^{\#}$ \\
\hline Leukocyte count, $\times 10^{9} / \mathrm{L}$ & $\begin{array}{c}5.8 \pm 2.8 \\
5.4(1.1-14.9)\end{array}$ & $\begin{array}{c}4.5 \pm 2.5 \\
3.9(0.6-14.6)\end{array}$ & $-27.5(-72.0$ to 139.3$)$ & $<0.001$ \\
\hline Neutrophil count, $\times 10^{9} / \mathrm{L}$ & $\begin{array}{c}3.8 \pm 2.3 \\
3.3(0.5-12.9)\end{array}$ & $\begin{array}{c}3.2 \pm 2.2 \\
2.6(0.3-12.5)\end{array}$ & $-22.7(-70.2$ to 258.6$)$ & $<0.001$ \\
\hline Lymphocyte count, $\times 10^{9} / \mathrm{L}$ & $\begin{array}{c}1.2 \pm 0.6 \\
\text { I.I }(0.3-3.1)\end{array}$ & $\begin{array}{c}0.7 \pm 0.4 \\
0.6(0.1-2.2)\end{array}$ & $-42.3(-90.3$ to 167.5$)$ & $<0.001$ \\
\hline Hemoglobin, g/dL & $\begin{array}{c}12.1 \pm 2.0 \\
11.9(6.7-17.4)\end{array}$ & $\begin{array}{c}11.9 \pm 1.9 \\
12.3(6.9-15.4)\end{array}$ & $-0.9(-35.2$ to 28.7$)$ & 0.203 \\
\hline Platelet count, $\times 10^{9} / \mathrm{L}$ & $\begin{array}{l}145.6 \pm 85.4 \\
124(24-479)\end{array}$ & $\begin{array}{l}102.4 \pm 64.6 \\
87(28-383)\end{array}$ & $-27.4(-83.2$ to 105.9$)$ & $<0.001$ \\
\hline NLR & $\begin{array}{c}3.6 \pm 2.5 \\
2.8(0.9-13.3)\end{array}$ & $\begin{array}{c}5.9 \pm 5.3 \\
4.3(0.9-28.7)\end{array}$ & $47.6(-81.9$ to 894.3$)$ & $<0.001$ \\
\hline PLR & $\begin{array}{c}132 \pm 79 \\
|| \mid(3 \mid-422)\end{array}$ & $\begin{array}{c}190 \pm \mid 80 \\
\mid 43(44-106 \mid)\end{array}$ & $30.2(-72.9$ to 740.7$)$ & 0.003 \\
\hline
\end{tabular}

Notes: Data are presented as mean \pm standard deviation and median (range); ${ }^{\#}$ The difference between pre- and post-SBRT immune parameters were evaluated using the Wilcoxon signed-rank test.

Abbreviations: NLR, neutrophil-to-lymphocyte ratio; PLR, platelet-to-lymphocyte ratio; SBRT, stereotactic body radiotherapy. 
Table 3 Prognostic Factors on Overall Survival by Cox Proportional-Hazards Model

\begin{tabular}{|c|c|c|c|c|c|c|}
\hline & \multicolumn{2}{|l|}{ UVA } & \multicolumn{2}{|c|}{ MVA in NLR Model } & \multicolumn{2}{|c|}{ MVA in PLR Model } \\
\hline & HR (95\% Cl) & $\mathbf{P}$ & HR (95\% Cl) & $\boldsymbol{P}$ & HR (95\% Cl) & $\mathbf{P}$ \\
\hline Age $>65$ years & $0.79(0.50-1.24)$ & 0.304 & & & & \\
\hline Female vs male & I.47 (0.87-2.48) & 0.151 & & & & \\
\hline Underlying liver disease & & & & & & \\
\hline HBV vs no & $0.79(0.50-1.25)$ & 0.309 & & & & \\
\hline HCV vs no & $1.00(0.62-1.62)$ & 0.987 & & & & \\
\hline CTP class $B$ vs $A$ & $2.07(1.28-3.33)$ & 0.003 & $2.35(1.27-4.37)$ & 0.007 & $2.48(1.38-4.44)$ & 0.002 \\
\hline Total GTV (per $10 \mathrm{cc}$ increase) & $1.002(0.996-1.008)$ & 0.566 & & & & \\
\hline Multiple tumors & $1.8 \mathrm{I}(1.09-3.03)$ & 0.023 & $1.99(1.08-3.68)$ & 0.028 & $1.71(0.93-3.15)$ & 0.083 \\
\hline Macrovascular invasion & $2.20(1.36-3.55)$ & 0.001 & I.5। (0.87-2.62) & 0.143 & $1.92(1.13-3.26)$ & 0.016 \\
\hline Extrahepatic spread & $3.35(1.92-5.85)$ & $<0.001$ & $1.68(0.91-3.08)$ & 0.097 & $1.61(0.85-3.04)$ & 0.141 \\
\hline Performance status $\geq 2$ & $2.01(1.17-3.46)$ & 0.012 & $0.77(0.40-1.50)$ & 0.443 & $1.14(0.61-2.15)$ & 0.676 \\
\hline AFP level $\geq 400 \mathrm{ng} / \mathrm{mL}$ & $1.67(1.06-2.65)$ & 0.029 & $1.72(1.00-2.95)$ & 0.051 & $1.59(0.94-2.69)$ & 0.081 \\
\hline BED $\geq 85.5$ Gy & $1.16(0.73-1.83)$ & 0.527 & & & & \\
\hline Prior treatment no. & & & & & & \\
\hline 0 & Reference & 0.552 & & & & \\
\hline $1-3$ & $1.30(0.78-2.16)$ & 0.311 & & & & \\
\hline$>3$ & $1.29(0.68-2.46)$ & 0.431 & & & & \\
\hline Pre-RT Hgb (per I g/dL increase) & $0.91(0.82-1.00)$ & 0.057 & $0.97(0.87-1.09)$ & 0.629 & $1.01(0.91-1.13)$ & 0.860 \\
\hline ASL & $2.33(1.44-3.76)$ & 0.001 & $1.01(0.54-1.90)$ & 0.971 & $1.67(0.99-2.84)$ & 0.056 \\
\hline Pre-RT NLR (per I-unit increase) & $1.20(1.10-1.30)$ & $<0.001$ & $1.24(1.12-1.38)$ & $<0.001$ & & \\
\hline Delta-NLR (per I-unit increase) & $1.11(1.05-1.18)$ & 0.001 & $1.10(1.02-1.18)$ & 0.011 & & \\
\hline Pre-RT PLR (per I0-unit increase) & $1.02(1.00-1.05)$ & 0.070 & & & $1.00(0.97-1.04)$ & 0.813 \\
\hline Delta-PLR (per 10-unit increase) & I.0I (0.99-I.02) & $0.40 \mathrm{I}$ & & & & \\
\hline
\end{tabular}

Abbreviations: AFP, $\alpha$-fetoprotein; ASL, acute severe lymphopenia; BED, biologically effective dose; Cl, confidence interval; CTP, Child-Turcotte-Pugh liver function scale; GTV, gross tumor volume; HBV, hepatitis B virus; HCV, hepatitis C virus; Hgb, hemoglobin; HR, hazard ratio; MVA, multivariable analysis; NLR, neutrophil-to-lymphocyte ratio; OS, overall survival; PLR, platelet-to-lymphocyte ratio; RT, radiotherapy; UVA, univariable analysis.

$<2.4$ and $\geq 2.4$ were 28.5 months ( $95 \%$ CI, $14.5-42.5$ ) and 6.0 months (95\% CI, 2.9-9.2), respectively $(p<0.001)$ (Figure 1A). In the subgroup analysis, there were no significant differences in 1-year OS between patients with dNLR $<1.3$ and dNLR $\geq 1.3$ ( $73.3 \%$ vs $92.9 \%, p=0.518)$ among 34 patients with pre-SBRT NLR $<2.4$ (Figure 1B). However, the 1-year OS and median OS were $44.1 \%$ and 10.7 months, respectively (95\% CI, 6.1-15.2) for patients with dNLR $<1.3$ compared with $18.4 \%$ and 3.8 months, respectively (95\% CI, 2.4-5.2) for those with dNLR $\geq 1.3$ ( $p=0.004$ ) (Figure 1C) among 59 patients with pre-SBRT $\mathrm{NLR} \geq 2.4$.
The overall survival (OS) by presence of prior liverdirected therapy and dNLR are as Supplementary Figure 1. Of note, for subgroup with history of prior surgery or RFA, patients with dNLR $<1.3$ had significantly longer OS than patients with $\mathrm{dNLR} \geq 1.3$ ( $p=0.016$ and 0.040 , respectively). There was a trend toward significance for OS among subgroup with prior TACE $(p=0.093)$. For subgroups without history of prior surgery, RFA or TACE, patients with dNLR $<1.3$ had numerically longer median OS than those with $\mathrm{dNLR} \geq 1.3$, but not statistically significant.

The median PFS time of the entire cohort was 6.2 months $(95 \% \mathrm{CI}, 4.8-7.7)$. After accounting for CTP 

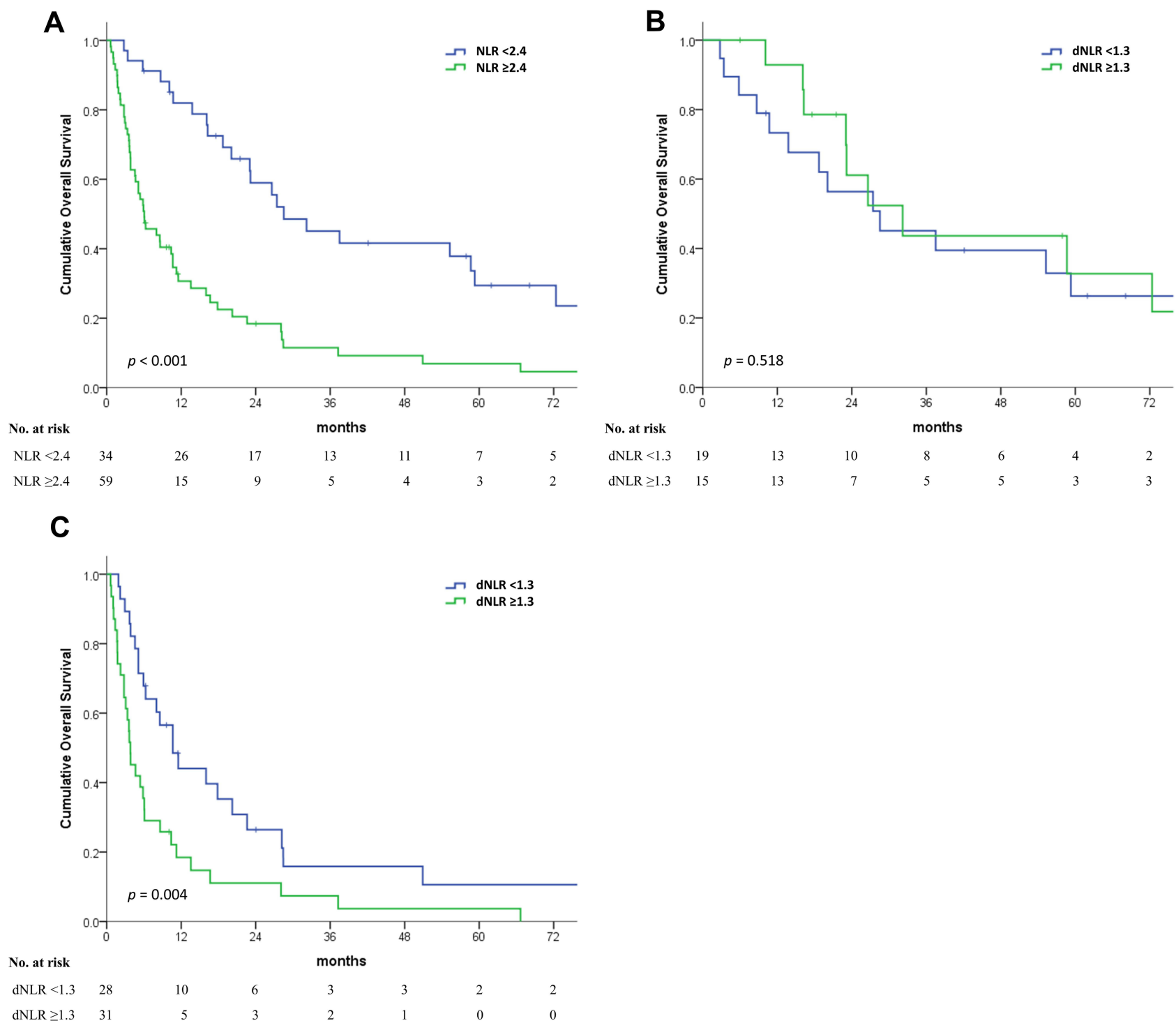

Figure I Survival groups based on pre-SBRT NLR and dNLR. Kaplan-Meier curve of overall survival (A) according to pre-SBRT NLR and subgroup comparisons among the (B) pre-SBRT NLR $<2.4$ cohort and (C) the pre-SBRT NLR $\geq 2.4$ cohort using the dNLR cut-off of I.3.

class, presence of multiple tumors, macrovascular invasion, extrahepatic spread, AFP level, BED, and pre-SBRT NLR, no statistically significant association existed between dNLR and PFS (HR, 0.96; 95\% CI, 0.881.05 per 1 -unit increase; $p=0.357$ ) (Supplementary Table 1). dPLR was not predictive of PFS in the univariable analysis.

\section{Association of Immune Parameters with Clinicopathologic Features, Dosimetry, and Liver Toxicity}

Relationships between dNLR and clinicopathologic features or dosimetry are detailed in Supplementary Table 2.
Only baseline CTP class was significantly associated with dNLR ( $p=0.007)$. CTP class B liver function patients had higher dNLR than CTP class A patients after SBRT (mean \pm SD, $5.1 \pm 7.3$ vs $1.2 \pm 3.1, p=0.004$ ). The median dNLR for CTP class A and B patients is 0.8 (range, -10.8 to 11.9) and 3.2 ( -6.2 to 23.6 ), respectively. Significant differences were observed only in post-SBRT NLR (mean \pm SD, 4.6 \pm 3.2 vs $9.0 \pm 7.7, p=0.004$ ) but not in pre-SBRT NLR between CTP class A and B patients ( $3.4 \pm 2.5$ vs $3.9 \pm 2.4$, $p=0.157$ ) (Figure 2A). The median pre- and post-SBRT NLR is 2.5 (range, 0.9-13.3) and $3.7(0.9-14.3)$ for CTP class A patients and 3.5 (1.2-11.7) and 6.0 (1.1-28.7) for CTP class B patients, respectively. There was no association between patients' baseline NLR and dNLR ( $p=0.945)$. 

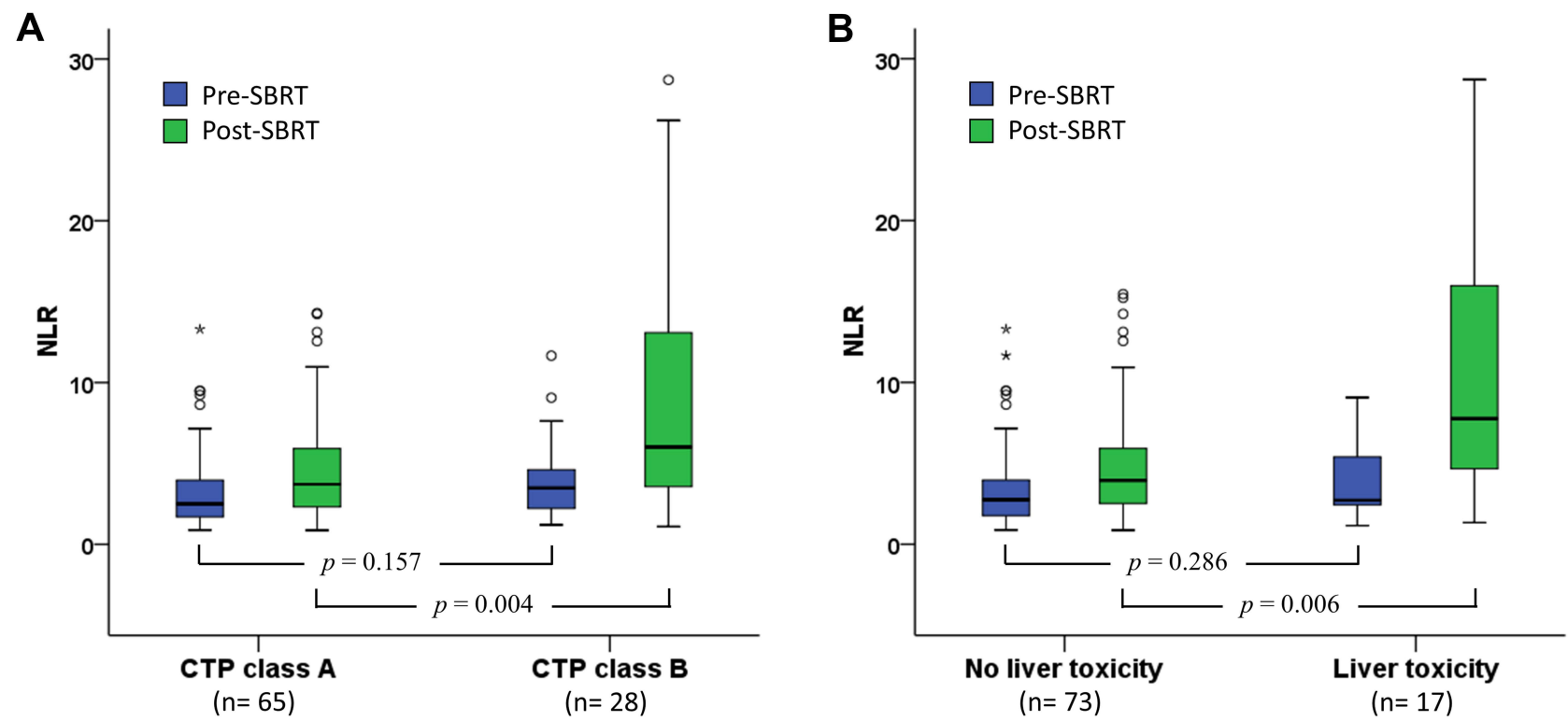

Figure 2 Box-whisker plot of peri-SBRT NLR changes in patients categorized according to (A) CTP class and (B) development of liver toxicity. Note only 90 patients were evaluable for liver toxicity. Extreme outliers and mild outliers were marked with an asterisk $(*)$ and a circle $(\mathrm{O})$ on the box-whisker plot, respectively.

For liver dosimetry, V5 had an association with dNLR $(p=0.066)$; however, this did not reach statistical significance.

Given the missing data, only 90 patients could be evaluated for liver toxicity, among whom 26 were CTP class B. Seventeen (18.9\%) patients developed liver toxicity, with higher risk for CTP class B group (38.5\% [10/ $26]$ vs $10.9 \%$ [7/64], $p=0.006$ ). Patients with liver toxicity had significantly higher dNLR than those without (mean $\pm \mathrm{SD}, 7.0 \pm 8.6$ vs $1.3 \pm 3.1, p=0.002$ ) (Figure $2 \mathrm{~B}$ ). The optimal dNLR cut-off value determined from the ROC curve for distinguishing the development of liver toxicity was 1.9 , with sensitivity of $76.5 \%$ and specificity of $67.1 \%$ (Supplementary Figure 2). The liver toxicity rate was $35.1 \%$ (13/37) for dNLR $\geq 1.9$ group, compared to $7.5 \%$ $(4 / 53)$ for dNLR $<1.9$ group $(p=0.002)$ (Figure 3). Higher dPLR was also noted for patients with liver toxicity, but not statistically significant ( $120 \pm 220$ vs $43 \pm 162, p=0.088)$.

\section{Discussion}

The key finding of this study was that both baseline level and the subsequent kinetics of the NLR are critical determinants of OS in a cohort of HCC patients treated with SBRT, illustrating the clinical significance of dynamic host immune status and response to radiotherapy. Higher dNLR was an independent inflammatory biomarker predictive of worse OS, especially among patients with a pretreatment NLR of $\geq 2.4$. dNLR was closely associated with baseline liver function and the presentation of liver toxicity in this patient population. To our knowledge, this is the largest study focused on peri-SBRT immune changes with respect to survival outcomes of HCC patients and the first study to evaluate their impacts on liver toxicity.

SBRT is well known as an extremely precise, highdose form of radiation therapy (RT) targeting a relatively small region encompassing the tumors while avoiding normal tissues from clinically intolerable radiation exposure. However, even with the favourable property of sharp dose fall-off and small field size compared to conventionally fractionated radiotherapy, SBRT still produces a certain degree of systemic effects on peripheral blood components during a course of limited fractionations for patients with lung, ${ }^{23}$ pancreas, ${ }^{18}$ and liver ${ }^{12,24}$ cancers. In vivo and in vitro investigations showed that human peripheral blood cells differ in their radiosensitivity, with lymphoid cells being extremely radiosensitive. ${ }^{25,26}$ The neutrophils and platelets are moderately radiosensitive, and erythrocytes are the most radioresistant hematologic cells. Recently, Wolfe et $\mathrm{al}^{13}$ reported that in patients with locally advanced pancreatic cancer treated with neoadjuvant therapy, lymphocyte counts showed a pronounced decline following conventional radiotherapy (36 or 50.4 Gy) compared to neutrophil counts (peri-RT percentage change, $-45.1 \%$ vs $-18.7 \%$ ), resulting in a marked increase in NLR by $99.7 \%$. In another study by Zhuang et al, a decrease in circulating blood cells was noted in 50 


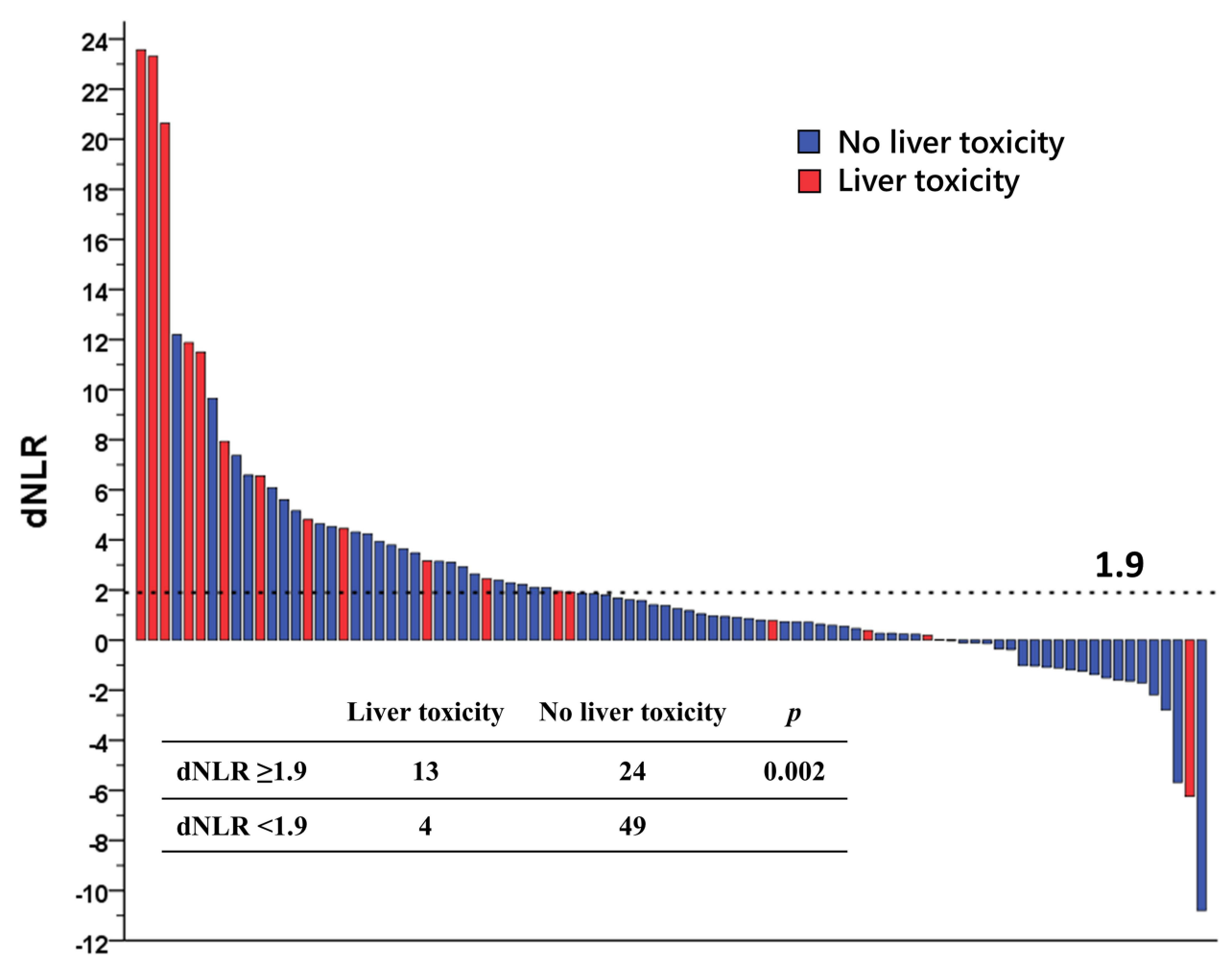

Figure 3 Waterfall plot of HCC patients ranked according to dNLR after SBRT. Patients with dNLR $\geq 1.9$ were more likely to present with liver toxicity than those with $\mathrm{dNLR}<1.9$.

patients receiving SBRT of $48-60$ Gy for small HCC. ${ }^{24}$ Although not addressed statistically, the lymphocyte counts had a larger decline (dropping by nearly half the pretreatment value) compared to other cells. We also found that the differential radiation response of immune cells leads to a significantly elevated NLR and PLR postSBRT. Accordingly, these data suggest that radiation of tumoricidal dose contributes to a potent pro-inflammatory systemic milieu for most patients (Figure 3), possibly significantly impacting host anticancer immune response and toxicity.

Previous studies have consistently demonstrated that a high baseline NLR was associated with adverse survival outcomes in many solid tumors. ${ }^{5-11,27}$ However, NLR appears as a dynamic measure of immunity, and some studies have suggested that decreasing NLR correlated with favourable outcomes in the setting of surgery for lung $^{28}$ or gastric ${ }^{29}$ cancers, chemotherapy for colorectal cancer, ${ }^{30}$ and immunotherapy for advanced solid tumors. ${ }^{31}$ For HCC, post-treatment NLR change was proposed to have superior discriminatory power for survival outcomes compared to baseline NLR in patients receiving $\mathrm{RFA}^{32}$ or sorafenib. ${ }^{33}$ Choi et al ${ }^{34}$ described the relationship between longitudinal NLR kinetics and clinical outcomes in nivolumab-treated patients with advanced HCC. They observed that NLR change at week 4 of nivolumab was related to treatment response, with decreased or unchanged NLR in responders to immunotherapy compared to the significantly increased NLR for patients with hyperprogressive disease. This ontreatment NLR kinetic (increased or decreased) was found to add the prognostic value to the baseline NLR in terms of predicting OS, especially those with high baseline $N L R \geq 3$. Moreover, the patients with baseline NLR $\geq 3$ and decreased NLR had comparable survival rates to those with baseline NLR $<3$ and increased NLR. Similar results were reported in RFA-treated HCC cases, using NLR at 1 month compared to the baseline. ${ }^{32}$ These results highlight the crucial role of immune response in determining the efficacy of antineoplastic therapies and may reverse the unfavourable pro-inflammatory background.

Here, the predictive value of baseline NLR and subsequent kinetics for survival were consistently observed. A high dNLR within 3 months could further stratify the OS only in patients with an elevated baseline NLR $(\geq 2.4)$. Neither PLR nor dPLR was predictive of OS or PFS in this cohort. Only one prior study has evaluated the predictive role of NLR and PLR in a dynamic setting in HCC 
patients treated with SBRT. $^{24}$ They focused on a population with small HCC and CTP class A liver function, among them only 50 patients had an available peritreatment immune profile (taken at median 7 days before and 10 days after SBRT) for analysis. They found that the low post-SBRT PLR $<263$ and limited NLR kinetics $<2.7$-fold were predictive of better OS and/or PFS, whereas baseline values of these parameters had no association with patients' survival. The discrepancy between that study and the present study may be due to differences in the composition of the enrolled populations.

Baseline liver reserve by CTP class is an established criterion in candidate selection and/or dose constraints in liver radiotherapy, where CTP A patients had better radiation tolerance and less liver toxicity compared to CTP B/C groups as shown in this cohort and most studies. The mechanisms by which SBRT confers liver toxicity and the causes for different toxicity results between liver function groups remain to be elucidated. A divergent activity of pro-inflammatory cytokines, such as tumor necrosis factor $\alpha$, was noted between HCC patients of different CTP scores at baseline and early during liver SBRT. ${ }^{35,36}$ This variable cytokine profile by liver function was suggested to mediate the differential radiation sensitivity of the liver and the development of liver toxicity, thereby affecting survival. In this cohort, the baseline CTP class was identified as the only predictor of dNLR after SBRT, and the difference in dNLR between CTP groups mainly arises from the post-SBRT change. It supports the presence of variable pro-inflammatory effects of radiation by liver function, probably through their divergent cytokine profile, contributing to the individual radiation tolerance. Given that dNLR is not predictive of PFS in this cohort, the OS difference between dNLR groups is likely attributable to their differential risk of liver toxicity. An augmented inflammation may be a key component for liver dysfunction after radiation, where dNLR of 1.9 is the optimal threshold for developing liver toxicity. In this regard, liver toxicity may be targetable with anti-inflammatory pharmaceuticals, and dNLR may be a monitoring tool for managing liver toxicity. ${ }^{37}$

This study had several limitations. First, given the retrospective nature of the study, peri-SBRT CBC data were not acquired at the same time point; it may add bias in measuring dNLR. Second, most included patients have underlying HBV infection and advanced disease; thus, the results may not be generalizable to the population of different aetiology or stage. Third, the long study duration causes the heterogeneity in the selection of radiotherapy machine and the technique to manage breathing motion. Fourth, the study was limited by relatively small numbers of patients in one institute; although this is the largest study on peri-SBRT immune changes. The effect of sequential treatments for recurrent tumors on the OS was also hard to estimate. Lastly, liver toxicity analysis was limited because of some missing data. Further validation in an independent cohort is warranted.

\section{Conclusion}

Our data demonstrate that liver SBRT produces a systemic pro-inflammatory effect as measured by dNLR in most HCC patients. Baseline NLR and dNLR can complementarily predict OS outcomes following SBRT for HCC. dNLR was associated with patients' baseline liver function that is linked to risk of liver toxicity, suggesting the role of augmented inflammation in the development of radiationrelated liver damage, where dNLR of 1.9 may be an optimal threshold. Additional studies are warranted to validate the prognostic value of dNLR and disclose their underlying mechanism in relation to liver toxicity.

\section{Abbreviations}

$\mathrm{ABC}$, active breathing control; ASL, acute severe lymphopenia; $\mathrm{BED}$, biologically effective dose; $\mathrm{CBC}$, complete blood count; CT, computed tomography; CTP, ChildTurcotte-Pugh; ITV, internal target volume; NLR, neutrophil-to-lymphocyte ratio; OS, overall survival; PFS, progression-free survival; PLR, platelet-to-lymphocyte ratio; RFA, radiofrequency ablation; RT, radiation therapy; SBRT, stereotactic body radiation therapy.

\section{Acknowledgments}

The authors thank the Cancer Registry Group of TriService General Hospital for the clinical data support.

\section{Funding}

This study was supported by study project of Tri-Service General Hospital (TSGH-D-110087 and TSGHD-110165). The authors thank the Cancer Registry Group of Tri-Service General Hospital for the clinical data support.

\section{Disclosure}

The authors report no conflicts of interest in this work. 


\section{References}

1. Pinero F, Dirchwolf M, Pessoa MG. Biomarkers in hepatocellular carcinoma: diagnosis, prognosis and treatment response assessment. Cells. 2020;9(6):1370. doi:10.3390/cells9061370

2. Grivennikov SI, Greten FR, Karin M. Immunity, inflammation, and cancer. Cell. 2010;140(6):883-899. doi:10.1016/j.cell.2010.01.025

3. Mantovani A, Allavena P, Sica A, Balkwill F. Cancer-related inflammation. Nature. 2008;454(7203):436-444. doi:10.1038/ nature 07205

4. Clarke SJ, Chua W, Moore M, et al. Use of inflammatory markers to guide cancer treatment. Clin Pharmacol Ther. 2011;90(3):475-478. doi:10.1038/clpt.2011.122

5. Liao W, Zhang J, Zhu Q, et al. Preoperative neutrophil-to-lymphocyte ratio as a new prognostic marker in hepatocellular carcinoma after curative resection. Transl Oncol. 2014;7(2):248-255. doi:10.1016/j. tranon.2014.02.011

6. Motomura T, Shirabe K, Mano Y, et al. Neutrophil-lymphocyte ratio reflects hepatocellular carcinoma recurrence after liver transplantation via inflammatory microenvironment. $J$ Hepatol. 2013;58 (1):58-64. doi:10.1016/j.jhep.2012.08.017

7. Fan W, Zhang Y, Wang Y, Yao X, Yang J, Li J. Neutrophil-tolymphocyte and platelet-to-lymphocyte ratios as predictors of survival and metastasis for recurrent hepatocellular carcinoma after transarterial chemoembolization. PLoS One. 2015;10(3):e0119312. doi:10.1371/journal.pone.0119312

8. Tian XC, Liu XL, Zeng FR, Chen Z, Wu DH. Platelet-to-lymphocyte ratio acts as an independent risk factor for patients with hepatitis $B$ virus-related hepatocellular carcinoma who received transarterial chemoembolization. Eur Rev Med Pharmacol Sci. 2016;20 (11):2302-2309.

9. Chu MO, Shen $\mathrm{CH}$, Chang TS, et al. Pretreatment inflammation-based markers predict survival outcomes in patients with early stage hepatocellular carcinoma after radiofrequency ablation. Sci Rep. 2018;8 (1):16611. doi:10.1038/s41598-018-34543-z

10. Bruix J, Cheng AL, Meinhardt G, Nakajima K, De Sanctis Y, Llovet J. Prognostic factors and predictors of sorafenib benefit in patients with hepatocellular carcinoma: analysis of two phase III studies. J Hepatol. 2017;67(5):999-1008. doi:10.1016/j. jhep.2017.06.026

11. Liu L, Gong Y, Zhang Q, Cai P, Feng L. Prognostic roles of blood inflammatory markers in hepatocellular carcinoma patients taking sorafenib. A systematic review and meta-analysis. Front Oncol. 2019;9:1557. doi:10.3389/fonc.2019.01557

12. Byun HK, Kim N, Park S, Seong J. Acute severe lymphopenia by radiotherapy is associated with reduced overall survival in hepatocellular carcinoma. Strahlenther Onkol. 2019;195(11):1007-1017. doi:10.1007/s00066-019-01462-5

13. Wolfe AR, Siedow M, Nalin A, et al. Increasing neutrophil-tolymphocyte ratio following radiation is a poor prognostic factor and directly correlates with splenic radiation dose in pancreatic cancer. Radiother Oncol. 2021;158:207-214. doi:10.1016/j. radonc.2021.02.035

14. Wahl DR, Stenmark MH, Tao Y, et al. Outcomes after stereotactic body radiotherapy or radiofrequency ablation for hepatocellular carcinoma. $J$ Clin Oncol. 2016;34(5):452-459. doi:10.1200/ JCO.2015.61.4925

15. Sapisochin G, Barry A, Doherty M, et al. Stereotactic body radiotherapy vs. TACE or RFA as a bridge to transplant in patients with hepatocellular carcinoma. An intention-to-treat analysis. $J$ Hepatol. 2017;67(1):92-99. doi:10.1016/j.jhep.2017.02.022

16. Sapir E, Tao Y, Schipper MJ, et al. Stereotactic body radiation therapy as an alternative to transarterial chemoembolization for hepatocellular carcinoma. Int J Radiat Oncol Biol Phys. 2018;100 (1):122-130. doi:10.1016/j.ijrobp.2017.09.001
17. Marciscano AE, Haimovitz-Friedman A, Lee P, et al. Immunomodulatory effects of stereotactic body radiation therapy: preclinical insights and clinical opportunities. Int $J$ Radiat Oncol Biol Phys. 2021;110(1):35-52. doi:10.1016/j.ijrobp.2019.02.046

18. Wild AT, Herman JM, Dholakia AS, et al. Lymphocyte-sparing effect of stereotactic body radiation therapy in patients with unresectable pancreatic cancer. Int J Radiat Oncol Biol Phys. 2016;94(3):571-579. doi:10.1016/j.ijrobp.2015.11.026

19. Lo CH, Lee HL, Hsiang CW, et al. Pretreatment neutrophil-tolymphocyte ratio predicts survival and liver toxicity in patients with hepatocellular carcinoma treated with stereotactic ablative radiation therapy. Int $J$ Radiat Oncol Biol Phys. 2021;109(2):474-484. doi:10.1016/j.ijrobp.2020.09.001

20. Lo CH, Liu MY, Lee MS, et al. Comparison between Child-TurcottePugh and albumin-bilirubin scores in assessing the prognosis of hepatocellular carcinoma after stereotactic ablative radiation therapy. Int J Radiat Oncol Biol Phys. 2017;99(1):145-152. doi:10.1016/j.ijrobp.2017.04.036

21. Huang WY, Jen YM, Lee MS, et al. Stereotactic body radiation therapy in recurrent hepatocellular carcinoma. Int $J$ Radiat Oncol Biol Phys. 2012;84(2):355-361. doi:10.1016/j.ijrobp.2011.11.058

22. Lencioni R, Llovet JM. Modified RECIST (mRECIST) assessment for hepatocellular carcinoma. Semin Liver Dis. 2010;30(1):52-60. doi:10.1055/s-0030-1247132

23. Navarro-Martin A, Galiana IL, Berenguer Frances MA, et al. Preliminary study of the effect of stereotactic body radiotherapy (SBRT) on the immune system in lung cancer patients unfit for surgery: immunophenotyping analysis. Int J Mol Sci. 2018;19 (12):3963. doi:10.3390/ijms 19123963

24. Zhuang Y, Yuan BY, Hu Y, et al. Pre/post-treatment dynamic of inflammatory markers has prognostic value in patients with small hepatocellular carcinoma managed by stereotactic body radiation therapy. Cancer Manag Res. 2019;11:10929-10937. doi:10.2147/ CMAR.S231901

25. Plowman PN. The effects of conventionally fractionated, extended portal radiotherapy on the human peripheral blood count. Int J Radiat Oncol Biol Phys. 1983;9(6):829-839. doi:10.1016/0360-3016(83)90008-1

26. Falcke SE, Ruhle PF, Deloch L, Fietkau R, Frey B, Gaipl US. Clinically relevant radiation exposure differentially impacts forms of cell death in human cells of the innate and adaptive immune system. Int J Mol Sci. 2018;19(11):3574. doi:10.3390/ijms19113574

27. Cupp MA, Cariolou M, Tzoulaki I, Aune D, Evangelou E, BerlangaTaylor AJ. Neutrophil to lymphocyte ratio and cancer prognosis: an umbrella review of systematic reviews and meta-analyses of observational studies. BMC Med. 2020;18(1):360. doi:10.1186/s12916-020-01817-1

28. Micaela R, Lucas C, Franco C, Federico C, Agustin D, David S. Dynamic perioperative variation of neutrophil-to-lymphocyte ratio as an independent prognosis factor following lobectomy for NSCLC. Updates Surg. 2021;73(4):1567-1574. doi:10.1007/s13304-02000936-x

29. Min KW, Kwon MJ, Kim DH, et al. Persistent elevation of postoperative neutrophil-to-lymphocyte ratio: a better predictor of survival in gastric cancer than elevated preoperative neutrophil-to-lymphocyte ratio. Sci Rep. 2017;7(1):13967. doi:10.1038/s41598-017-13969-x

30. Chua W, Charles KA, Baracos VE, Clarke SJ. Neutrophil/lymphocyte ratio predicts chemotherapy outcomes in patients with advanced colorectal cancer. Br J Cancer. 2011;104(8):1288-1295. doi:10.1038/ bjc. 2011.100

31. Ameratunga M, Chenard-Poirier M, Moreno Candilejo I, et al. Neutrophil-lymphocyte ratio kinetics in patients with advanced solid tumours on phase I trials of PD-1/PD-L1 inhibitors. Eur J Cancer. 2018;89:56-63. doi:10.1016/j.ejca.2017.11.012

32. Dan J, Zhang Y, Peng Z, et al. Postoperative neutrophil-tolymphocyte ratio change predicts survival of patients with small hepatocellular carcinoma undergoing radiofrequency ablation. PLoS One. 2013;8(3):e58184. doi:10.1371/journal.pone.0058184 
33. Hong YM, Yoon KT, Hwang TH, Heo J, Woo HY, Cho M. Changes in the neutrophil-to-lymphocyte ratio predict the prognosis of patients with advanced hepatocellular carcinoma treated with sorafenib. Eur $J$ Gastroenterol Hepatol. 2019;31(10):1250-1255. doi:10.1097/ MEG.0000000000001405

34. Choi WM, Kim JY, Choi J, et al. Kinetics of the neutrophil-lymphocyte ratio during PD-1 inhibition as a prognostic factor in advanced hepatocellular carcinoma. Liver Int. 2021;41(9):2189-2199. doi:10.1111/liv.14932

35. Cousins MM, Morris E, Maurino C, et al. TNFR1 and the TNFalpha axis as a targetable mediator of liver injury from stereotactic body radiation therapy. Transl Oncol. 2021;14(1):100950. doi:10.1016/j. tranon.2020.100950
36. Ng SSW, Zhang H, Wang L, Citrin D, Dawson LA. Association of pro-inflammatory soluble cytokine receptors early during hepatocellular carcinoma stereotactic radiotherapy with liver toxicity. NPJ Precis Oncol. 2020;4:17. doi:10.1038/s41698-020-0124-z

37. Najar Nobari N, Shahidi Dadras M, Nasiri S, Abdollahimajd F, Gheisari M. Neutrophil/platelet to lymphocyte ratio in monitoring of response to TNF-alpha inhibitors in psoriatic patients. Dermatol Ther. 2020;33(4):e13457. doi:10.1111/dth.13457

\section{Publish your work in this journal}

The Journal of Hepatocellular Carcinoma is an international, peerreviewed, open access journal that offers a platform for the dissemination and study of clinical, translational and basic research findings in this rapidly developing field. Development in areas including, but not limited to, epidemiology, vaccination, hepatitis therapy, pathology and molecular tumor classification and prognostication are all considered for publication. The manuscript management system is completely online and includes a very quick and fair peer-review system, which is all easy to use. Visit http://www.dovepress.com/ testimonials.php to read real quotes from published authors.

Submit your manuscript here: https://www.dovepress.com/journal-of-hepatocellular-carcinoma-journal 